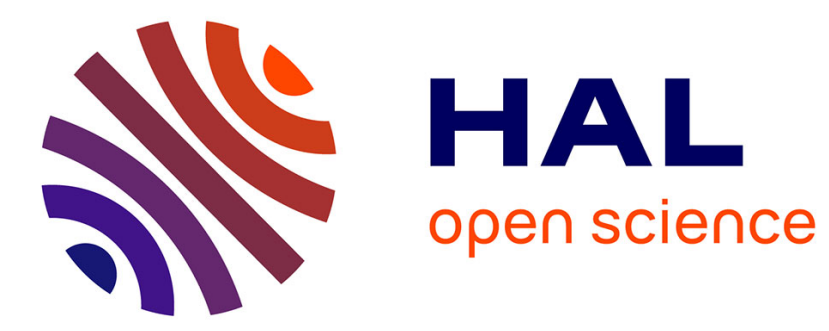

\title{
Influence de la contre-réaction thermique sur l'impédance de sortie des transistors MOS à canaux courts
}

\author{
P. Rossel, M. Gamboa, Henri Tranduc, H. Martinot
}

\section{- To cite this version:}

P. Rossel, M. Gamboa, Henri Tranduc, H. Martinot. Influence de la contre-réaction thermique sur l'impédance de sortie des transistors MOS à canaux courts. Revue de Physique Appliquée, 1979, 14 (11), pp.911-919. 10.1051/rphysap:019790014011091100 \& jpa-00244679

\section{HAL Id: jpa-00244679 https://hal.science/jpa-00244679}

Submitted on 1 Jan 1979

HAL is a multi-disciplinary open access archive for the deposit and dissemination of scientific research documents, whether they are published or not. The documents may come from teaching and research institutions in France or abroad, or from public or private research centers.
L'archive ouverte pluridisciplinaire HAL, est destinée au dépôt et à la diffusion de documents scientifiques de niveau recherche, publiés ou non, émanant des établissements d'enseignement et de recherche français ou étrangers, des laboratoires publics ou privés. 


\title{
Influence de la contre-réaction thermique sur l'impédance de sortie des transistors MOS à canaux courts
}

\author{
P. Rossel, M. Gamboa, H. Tranduc and H. Martinot \\ Laboratoire d'Automatique et d'Analyse des Systèmes, 7, avenue du Colonel-Roche, 31400 Toulouse, France
}

(Reçu le 8 juin 1979, révisé le 20 juillet 1979, accepté le 25 juillet 1979)

\begin{abstract}
Résumé. - Les propriétés de l'admittance de sortie en régime de saturation des transistors MOS à canaux courts sont étudiées dans le domaine des basses fréquences. Les signes et les amplitudes des parties réelles et imaginaires de l'admittance dépendent des conditions de polarisation. Le diagramme complexe est interprété par l'association des mécanismes électriques et de l'effet de contre-réaction thermique. Une formulation théorique de cette admittance est proposée et comparée aux résultats expérimentaux. On en déduit une méthode de détermination de l'impédance thermique et du schéma équivalent électrique du transistor.
\end{abstract}

\begin{abstract}
The output admittance properties of the short channel MOS transistors, operating beyond pinch-off, are studied in the low frequency range. Both the signs and amplitudes of the real and imaginary parts of this admittance depend upon the bias condition. The properties of the complex diagram are accounted for by taking into account the internal feedback between the electrical mechanisms and the thermal phenomena. A theoretical expression of the admittance is derived and compared with the experimental results. A method for determining the thermal impedance and the equivalent circuit of the MOST is proposed.
\end{abstract}

1. Introduction. - Dans les transistors MOS dont la distance source-drain est supérieure à dix microns environ, la conductance de sortie est toujours positive, quelles que soient les valeurs des tensions de polarisation de grille et de drain, du courant de drain et quel que soit le régime de fonctionnement, saturé ou non saturé. Les propriétés de la conductance de sortie en régime de saturation, ou de pincement, pour cette famille de transistors MOS, communément appelés les transistors à canal long, ont fait l'objet de nombreuses publications. L'interprétation de l'existence d'une conductance de sortie finie, dépendante des tensions de polarisation, est liée à l'existence d'une zone de charge d'espace près du drain qui entraîne par effet électrostatique une réduction de la longueur effective du canal. Les diverses analyses se différencient par la caractérisation de cette zone de charge d'espace : représentation unidimensionnelle [1] ou bidimensionnelle [2], prise en compte de la charge des porteurs mobiles [3], définition des conditions aux limites sur le champ électrique et le potentiel électrostatique $[4,5,6]$.

Lorsque la distance source-drain est inférieure à dix microns environ, il apparaît que la conductance de sortie en régime statique peut présenter des valeurs négatives quand le transistor MOS, appelé dans ce cas transistor à canal court, fonctionne à des niveaux élevés de courant de drain (Fig. 1). Une interprétation de ce phénomène a été proposée [7] : elle prend en compte la diminution de la mobilité des porteurs de la couche d'inversion qui constitue le canal, sous l'effet de l'échauffement interne du transistor dû à la puissance continue que ce dernier dissipe.

Afin de mieux caractériser ce phénomène, nous étudions les propriétés de l'admittance de sortie des transistors à canal court en régime de saturation et dans le domaine des basses fréquences.

Les propriétés expérimentales de cette admittance seront tout d'abord décrites : existence de conductances de sortie positive ou négative et de susceptances de type inductif ou capacitif. Une analyse prenant en compte l'effet de réaction entre les mécanismes thermiques et les propriétés électriques en régime dynamique de basses fréquences et de petits signaux, sera ensuite développée et permettra d'expliquer les propriétés fonctionnelles de l'admittance de sortie. Enfin, sur la base de cette analyse, on proposera une méthode de détermination de l'impédance thermique complexe de l'ensemble transistor-boîtier et un schéma équivalent électrique du transistor sera établi. 


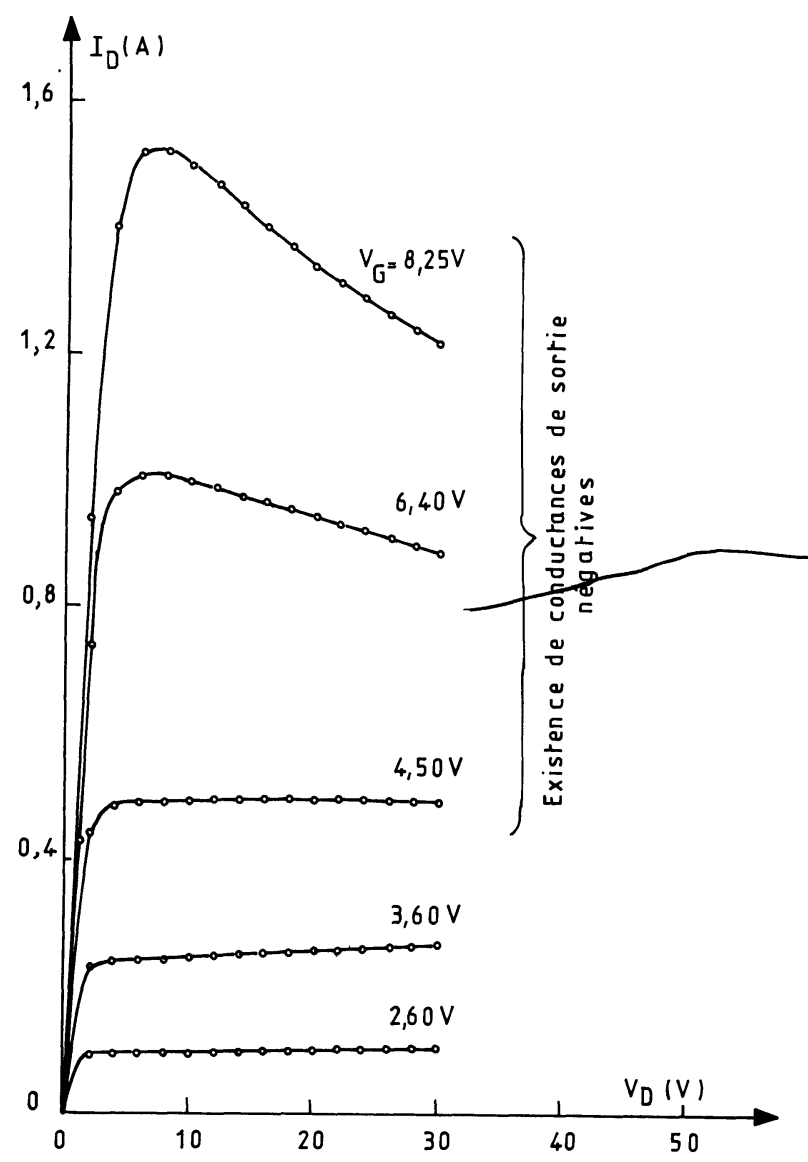

Fig. 1. - Mise en évidence de l'existence de conductances de sortie positive ou négative sur un transistor à canal court fonctionnant en régime statique ( $T$ boîtier $=20^{\circ} \mathrm{C}$ ).

[DC characteristics of a short channel MOS transistor showing the existence of either positive or negative output conductance $\left(T\right.$ case $\left.=20^{\circ} \mathrm{C}\right)$.]

2. Propriétés expérimentales. - Les dispositifs étudiés sont des transistors interdigités dont le canal vertical (V-MOS) a $2 \mu \mathrm{m}$ environ de longueur et une largeur égale à $3 \mathrm{~cm}$. La puce de silicium de $1 \mathrm{~mm}^{2}$ de surface est fixée sur une embase en cuivre de type TO 3. Cette dernière est montée sur un radiateur infini refroidi par fluide et sa température est imposée quelles que soient les conditions de fonctionnement.

L'admittance de sortie en petits signaux est mesurée au moyen d'un système de détection synchrone (Fig. 2). La structure est polarisée en continu par la tension drain $V_{\mathrm{D}}$ et la tension grille $V_{\mathrm{G}}$ et est traversée par un courant statique $I_{\mathrm{D}}$.

Une tension alternative de faible amplitude ( $<100 \mathrm{mV}$ efficaces) $v(\omega)$ est superposée à la tension de drain par l'ensemble générateur de puissancetransformateur large bande-pont de résistances $R_{1}, R_{2}$. La mesure des composantes en phase et en quadrature $\mathrm{du}$ courant alternatif $i(\omega)$ résultant de l'excitation $v(\omega)$ (Fig. 3) est effectuée au moyen de l'ensemble- $\mathbf{R}_{4}$ détecteur synchrone. On obtient ainsi les parties réelle et imaginaire de l'admittance de sortie $\mathrm{Y}(\omega)$

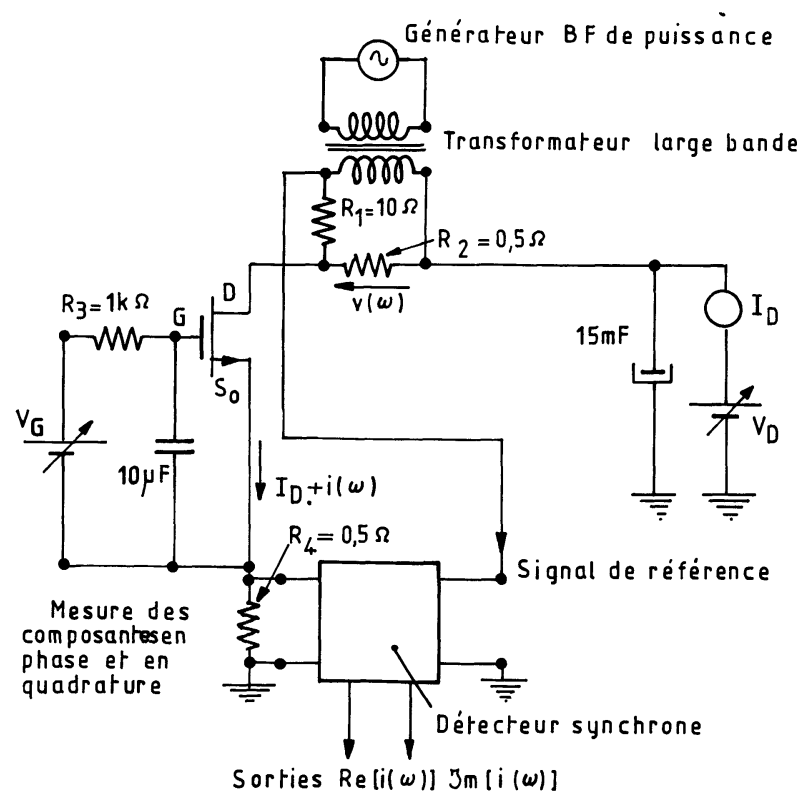

Fig. 2. - Dispositif de mesure de l'admittance de sortie.

[The output admittance measurement system.]

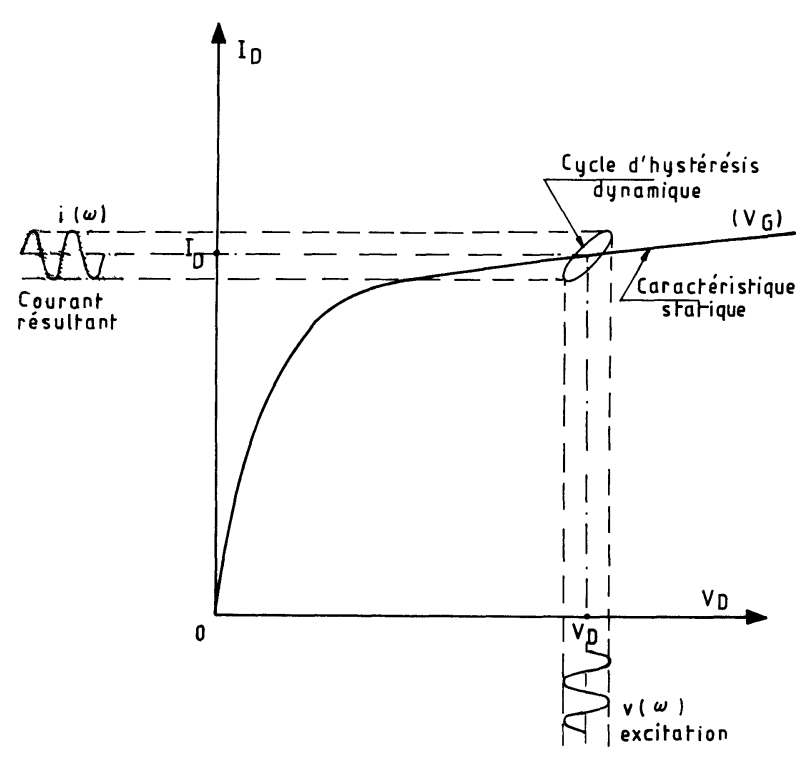

Fig. 3. - Principe de mesure de l'admittance de sortie.

[Principle of the output admittance determination.]

qui est définie par le rapport $i(\omega) / v(\omega) ; \omega$ est la pulsation des signaux d'excitation à la fréquence $f$. Les mesures sont effectuées entre $10 \mathrm{~Hz}$ et $100 \mathrm{kHz}$ ainsi qu'en régime statique $(f=0)$ et on trace dans le plan de Nyquist les variations de la partie imaginaire Im (Y) en fonction de la partie réelle $\operatorname{Re}(\mathrm{Y})$ pour diverses valeurs de la fréquence.

Les propriétés observées expérimentalement sont les suivantes :

(i) Pour une valeur fixée de la tension de drain $V_{D}$, le courant $I_{\mathrm{D}}$ étant considéré comme un paramètre, 
le diagramme complexe a une partie imaginaire négative (Fig. 4), c'est-à-dire que l'impédance de sortie est du type inductif, pour les valeurs du courant de drain $I_{\mathrm{D}}$ comprises entre la valeur nulle et une valeur critique que nous appelerons $I_{C}$ (voisine de $250 \mathrm{~mA}$ pour les structures étudiées). Cette partie imaginaire est nulle en régime statique et tend également vers une valeur nulle en hautes fréquences (supérieures à $50 \mathrm{kHz}$ ). Lorsque le courant de drain est supérieur à la valeur $I_{C}$ la partie imaginaire est positive ; l'impédance de sortie a un comportement capacitif (Fig. 5).

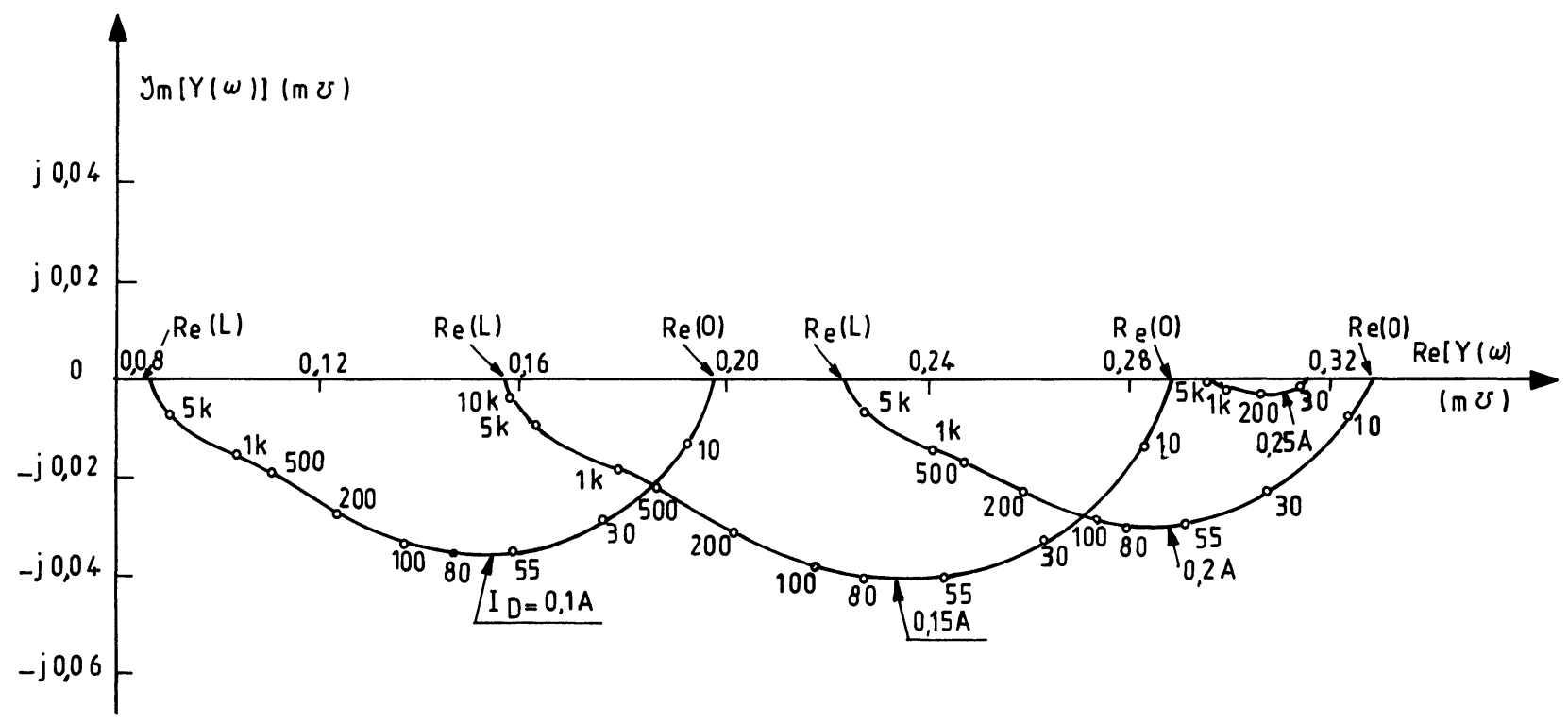

Fig. 4. - Influence du courant de drain sur le diagramme complexe d'admittance de sortie ; cas des parties imaginaires négatives $\left(I_{\mathrm{D}}<I_{\mathrm{C}}\right)$. $V_{\mathrm{D}}=20 \mathrm{~V} ; T_{\mathrm{a}}=20^{\circ} \mathrm{C}$. La fréquence est le paramètre en hertz ou en kilohertz (K).

[Influence of the drain current on the complex diagram of the output admittance $\left(I_{\mathrm{D}}<I_{\mathrm{C}}\right):$ the imaginary parts are negative. $V_{\mathrm{D}}=20 \mathrm{~V}$. $T_{\mathrm{a}}=20^{\circ} \mathrm{C}$. Frequency as parameter : hertz or kilohertz $\left.(\mathrm{K}) \cdot\right]$

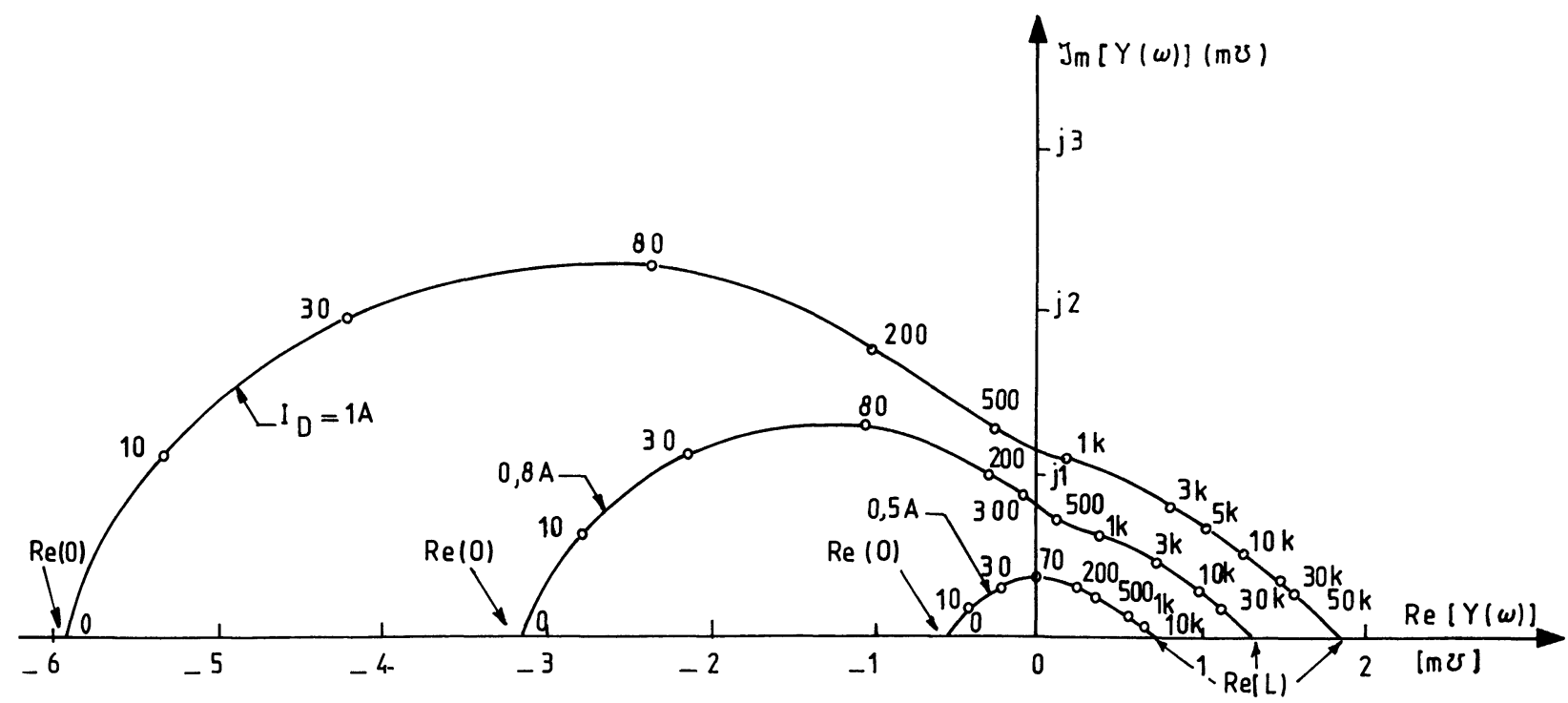

Fig. 5. - Influence du courant de drain sur le diagramme complexe d'admittance de sortie; cas des parties imaginaires positives $\left(I_{\mathrm{D}}>I_{\mathrm{C}}\right)$. $V_{\mathrm{D}}=20 \mathrm{~V} ; T_{\mathrm{a}}=20^{\circ} \mathrm{C}$. La fréquence est le paramètre.

[Influence of the drain current on the complex diagram of the output admittance $\left(I_{\mathrm{D}}>I_{\mathrm{C}}\right):$ the imaginary parts are positive. $V_{\mathrm{D}}=20 \mathrm{~V}$. $T_{\mathrm{a}}=20^{\circ} \mathrm{C}$. Frequency as parameter.] 


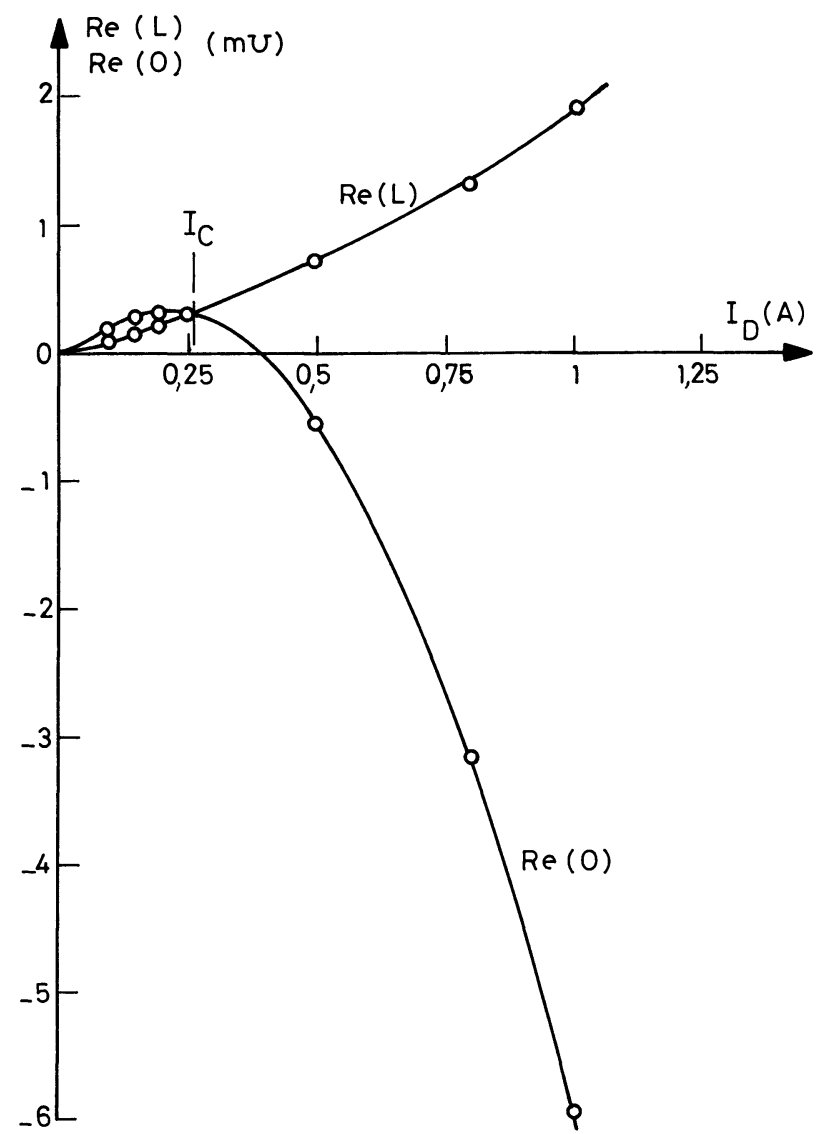

Fig. 6. - Variations expérimentales de la conductance de sortie en fonction du courant de drain : régime statique $\operatorname{Re}(0)$ et régime de hautes fréquences $\operatorname{Re}(\mathrm{L}) . V_{\mathrm{D}}=20 \mathrm{~V} ; T_{\mathrm{a}}=20^{\circ} \mathrm{C}$.

[Output conductance - DC conditions $\operatorname{Re}(0)$ and HF conditions $\operatorname{Re}(\mathrm{L})$ - as a function of the drain current $V_{\mathrm{D}}=20 \mathrm{~V}$; $\left.T_{\mathrm{a}}=20^{\circ} \mathrm{C}.\right]$ (ii) Quelles que soient les valeurs du courant ou de la tension de drain, le maximum de la partie imaginaire qui sera noté $\mathrm{Jm}_{\max }$ se produit pour la même valeur de la fréquence.

(iii) La partie réelle peut être positive ou négative : aux fréquences élevées $(f>50 \mathrm{kHz})$ elle est toujours positive et sera appelée $\operatorname{Re}(\mathrm{L})$; à la fréquence nulle, elle est positive pour les faibles valeurs du courant et peut devenir négative à la condition que ce dernier soit très supérieur à la valeur critique $I_{\mathrm{C}}$; elle sera appelée $\operatorname{Re}(0)$. La figure 6 représente les variations de ces parties réelles $\operatorname{Re}(0)$ et $\operatorname{Re}(\mathrm{L})$ en fonction du courant de drain.

(iv) Lorsque le courant de drain est maintenu constant et que la tension de drain augmente (Fig. 7), on observe une translation du diagramme dans la direction des parties réelles négatives, la partie imaginaire restant indépendante de la tension $V_{\mathbf{D}}$.

3. Expression de l'admittance de sortie. - L'origine des phénomènes observés doit être recherchée dans un couplage entre les mécanismes électriques et thermiques dans le canal du transistor. De façon générale, rappelons que lorsqu'on applique la théorie des dipôles ou des quadripôles pour établir le schéma équivalent d'un dispositif électronique, on suppose d'ordinaire que la température des éléments constitutifs du dipôle ou du quadripôle ne dépend pas du temps ou de la fréquence. Toutefois, si les inverses des constantes de temps thermiques associées à ces éléments sont du même ordre de grandeur que la fréquence des signaux d'excitation, cette hypothèse n'est plus valable [8]. Dans ces conditions, il en résulte

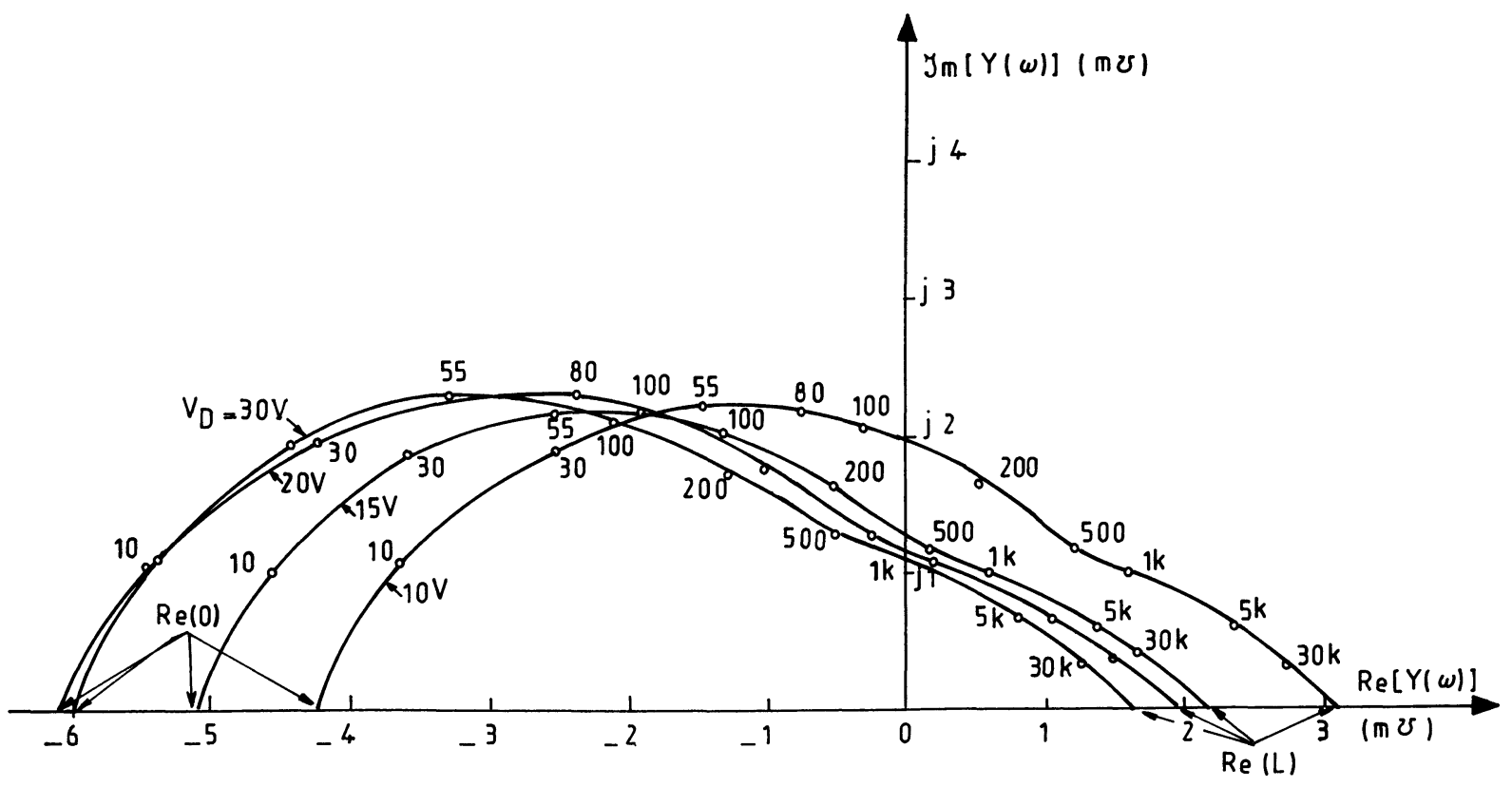

Fig. 7. - Influence de la tension de drain sur le diagramme complexe d'admittance de sortie. $I_{\mathrm{D}}=1 \mathrm{~A} ; T_{\mathrm{a}}=20^{\circ} \mathrm{C}$. La fréquence est le paramètre.

[Influence of the drain voltage on the output admittance diagram. $I_{\mathrm{D}}=1 \mathrm{~A} ; T_{\mathrm{a}}=20^{\circ} \mathrm{C}$. Frequency as parameter.] 
une interaction de type électrique-thermique ou encore une contre-réaction thermique, qui apparaît principalement en basses fréquences et qui modifie les paramètres du schéma équivalent. Cet effet ne doit pas être confondu avec l'effet classique de la dépendance en température des éléments constitutifs du dipôle ou du quadripôle.

Dans le cas du transistor MOS, considéré dans le cadre de cette étude comme un dipôle dont les électrodes sont la source et le drain, l'expression de l'admittance de sortie sera obtenue en associant la relation de définition du courant de drain en régime de petits signaux à celle qui exprime l'échauffement dans le formalisme de l'impédance thermique complexe [9].

L'hypothèse de petits signaux permet la linéarisation du courant de drain, de la tension drain-source et de la température de cristal. A tension grille-source, $V_{\mathrm{G}}$, constante, ces quantités s'écrivent respectivement :

$$
\begin{aligned}
I_{\mathrm{D}}\left(T, V_{\mathrm{D}}, \omega\right) & =I_{\mathrm{D}}+i(\omega) \\
V_{\mathrm{D}}(\omega) & =V_{\mathrm{D}}+v(\omega) \\
T(\omega) & =T+\theta(\omega)
\end{aligned}
$$

où $I_{\mathrm{D}}, V_{\mathrm{D}}, T$ sont les valeurs du courant et de la tension drain, et de la température de canal, imposées par les conditions de polarisation statiques. $\theta(\omega)$ est la variation de température créée par les conditions d'excitation dynamiques.

Les relations qui permettent de calculer l'admittance de sortie sont d'une part l'expression de la différentielle totale du courant de drain :

$$
i(\omega)=\frac{\partial I_{\mathrm{D}}}{\partial V_{\mathrm{D}}} \cdot v(\omega)+\frac{\partial I_{\mathrm{D}}}{\partial T} \cdot \theta(\omega)
$$

et d'autre part, la relation liant l'échauffement à la puissance dynamique $p(\omega)$ :

$$
\theta(\omega)=Z_{\mathrm{th}}(\omega) \cdot p(\omega)
$$

dans laquelle la puissance dynamique s'exprime par :

$$
p(\omega)=i(\omega) \cdot V_{\mathbf{D}}+v(\omega) \cdot I_{\mathbf{D}}
$$

$Z_{\mathrm{th}}(\omega)$ représente l'impédance thermique complexe - entre le canal du transistor et le radiateur infini.

Compte tenu de (4), (5), (6), l'admittance de sortie peut s'écrire sous la forme :

$$
\begin{aligned}
Y(\omega)=\frac{1}{1-Z_{\mathrm{th}}(\omega) \cdot V_{\mathrm{D}} \cdot \frac{\partial I_{\mathrm{D}}}{\partial T}} \times \\
\times\left[\frac{\partial I_{\mathrm{D}}}{\partial V_{\mathrm{D}}}+Z_{\mathrm{th}}(\omega) \cdot \frac{\partial I_{\mathrm{D}}}{\partial T} \cdot I_{\mathrm{D}}\right] .
\end{aligned}
$$

On peut remarquer que cette analyse est générale et est applicable à d'autres composants semiconducteurs pouvant être représentés par un dipôle dont les éléments constitutifs dépendent de la température.
Dans le deuxième membre de la relation (7) le terme $\partial I_{\mathrm{D}} / \partial V_{\mathrm{D}}$ représente la conductance de sortie du transistor MOS liée à l'effet électrostatique de la tension de drain, qui est généralement étudiée pour décrire les propriétés en régime de saturation des transistors MOS. Par ailleurs, le terme $\partial I_{\mathrm{D}} / \partial T$ est le coefficient de température du courant de drain. La quantité $Z_{\mathrm{th}}(\omega) \cdot V_{\mathrm{D}} \cdot \frac{\partial I_{\mathrm{D}}}{\partial T}$ est négligeable devant l'unité pour la plupart des transistors MOS. Il vient :

$$
Y(\omega) \simeq \frac{\partial I_{\mathrm{D}}}{\partial V_{\mathrm{D}}}+Z_{\mathrm{th}}(\omega) \cdot \frac{\partial I_{\mathrm{D}}}{\partial T} \cdot I_{\mathrm{D}}
$$

L'admittance de sortie du transistor est la somme de l'admittance du transistor idéal dans lequel les effets thermiques ne sont pas pris en compte et d'une admittance égale au produit de l'impédance thermique, du coefficient de température et du courant de drain.

4. Interprétation des résultats et conséquences. L'analyse théorique précédente permet d'une part de comprendre les propriétés expérimentales et d'autre part de déterminer l'impédance thermique du composant et son schéma équivalent.

4.1 INTERPRÉTATION DES RÉSUltats. - (i) Les valeurs limites de l'admittance de sortie en régime statique et en régime de hautes fréquences s'identifient respectivement aux admittances suivantes :

$$
\begin{aligned}
& \operatorname{Re}(0)=Y(\omega=0)=\frac{\partial I_{\mathrm{D}}}{\partial V_{\mathrm{D}}}+R_{\mathrm{th}} \cdot \frac{\partial I_{\mathrm{D}}}{\partial T} \cdot I_{\mathrm{D}} \\
& \operatorname{Re}(\mathrm{L})=Y(\omega \rightarrow \infty)=\frac{\partial I_{\mathrm{D}}}{\partial V_{\mathrm{D}}}
\end{aligned}
$$

où $R_{\text {th }}$ est la partie réelle de l'impédance thermique c'est-à-dire la résistance thermique entre la région du canal et le radiateur. Ces admittances sont réelles. La deuxième $\operatorname{Re}(\mathrm{L})$ est toujours positive. Quant à la quantité $\operatorname{Re}(0)-\operatorname{Re}(\mathrm{L})$, elle peut être positive ou négative suivant le signe du coefficient de température [11].

Ceci explique qu'en hautes fréquences, la résistance de sortie soit positive alors qu'en régime statique, celle-ci peut présenter l'un ou l'autre des signes comme nous l'avons observé expérimentalement (Fig. 6).

(ii) La partie imaginaire a le signe contraire de celui du coefficient thermique et peut également être négative ou positive.

(iii) De façon plus quantitative, le coefficient de température des transistors étudiés a été mesuré en utilisant une méthode de microscopie infra-rouge [10] qui permet de déterminer directement les variations de température du canal du MOST. La figure 8 représente les évolutions de ce coefficient $\partial I_{\mathrm{D}} / \partial T$ en fonction du courant de drain. Les variations observées 


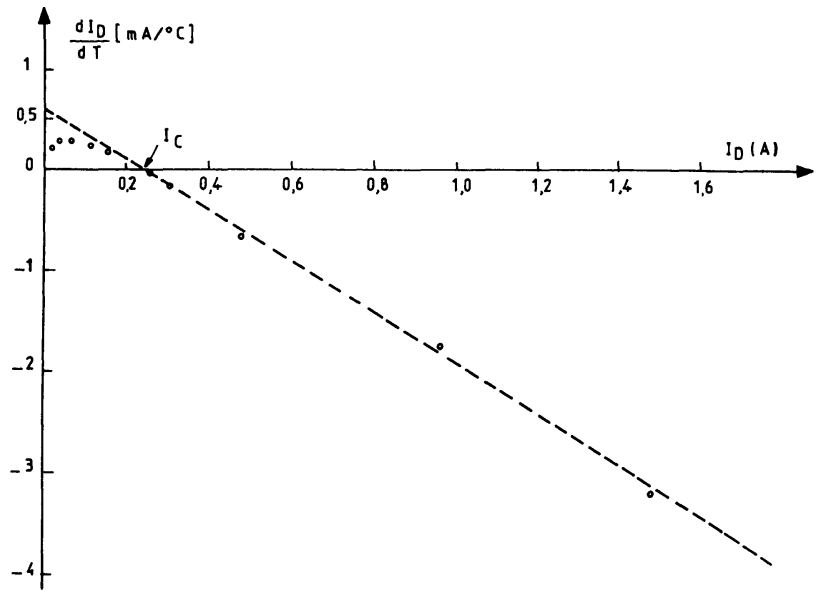

Fig. 8. - Variations du coefficient thermique en fonction du courant de drain. $\circ$ Expérience. --------- Approximation utilisée.

[Variations of the thermal coefficient as a function of the drain current. • Experimental values. --.---.-- Analytical approximation.]

sont similaires à celles régissant le comportement de tous les transistors MOS : pour les faibles valeurs du courant, le coefficient est positif, et est essentiellement contrôlé par les variations thermiques de la tension de seuil du transistor. A fort niveau de courant, le coefficient est négatif et dépend principalement de l'effet de la température sur la vitesse des porteurs du canal [3, 15].

En régime de pincement et au premier ordre, il est indépendant de la température de cristal

$$
\left(50^{\circ} \mathrm{C}<T<150^{\circ} \mathrm{C}\right)
$$

et peut être approximé par la relation :

$$
\frac{\partial I_{\mathrm{D}}}{\partial T}=-k\left(I_{\mathrm{D}}-I_{\mathrm{C}}\right)
$$

où $k$ est un coefficient fixé par la dépendance thermique de la vitesse (égal à $2,5 \times 10^{-3} d^{-1}$ pour les transistors étudiés), et $I_{\mathrm{C}}$ la valeur du courant pour laquelle les effets antagonistes, liés à la tension de seuil et à la vitesse, se compensent et le coefficient de température s'annule.

Par suite, compte tenu de (9), (10), (11), d'une part et de (8) et (11) d'autre part, il apparaît que :

- l'amplitude réelle du diagramme d'admittance $\operatorname{Re}(\mathrm{L})-\operatorname{Re}(0)$ est une fonction parabolique du courant de drain :

$\operatorname{Re}(\mathrm{L})-\operatorname{Re}(0)=k \cdot R_{\mathrm{th}} \cdot\left[\left(I_{\mathrm{D}}-\frac{I_{\mathrm{C}}}{2}\right)^{2}-\left(\frac{I_{\mathrm{C}}}{2}\right)^{2}\right]$,

- le maximum de la partie imaginaire de ce diagramme est aussi une fonction parabolique du courant de drain :

$$
\begin{aligned}
J_{\max }=\operatorname{Jm}\left(Z_{\mathrm{th}}(\omega)\right. & \left.\left.\right|_{\max }\right) \cdot(-k) \times \\
\times & {\left[\left(I_{\mathrm{D}}-\frac{I_{\mathrm{C}}}{2}\right)^{2}-\left(\frac{I_{\mathrm{C}}}{2}\right)^{2}\right], }
\end{aligned}
$$

- le diagramme d'admittance translaté par la quantité $\operatorname{Re}(\mathrm{L})$ est indépendant de la tension de drain :

$$
Y(\omega)-\operatorname{Re}(\mathrm{L})=-k \cdot Z_{\mathrm{th}}(\omega) \cdot I_{\mathrm{D}} \cdot\left(I_{\mathrm{D}}-I_{\mathrm{C}}\right)
$$

toutes ces propriétés sont bien observées expérimentalement comme le montrent les figures 7 et 9 .

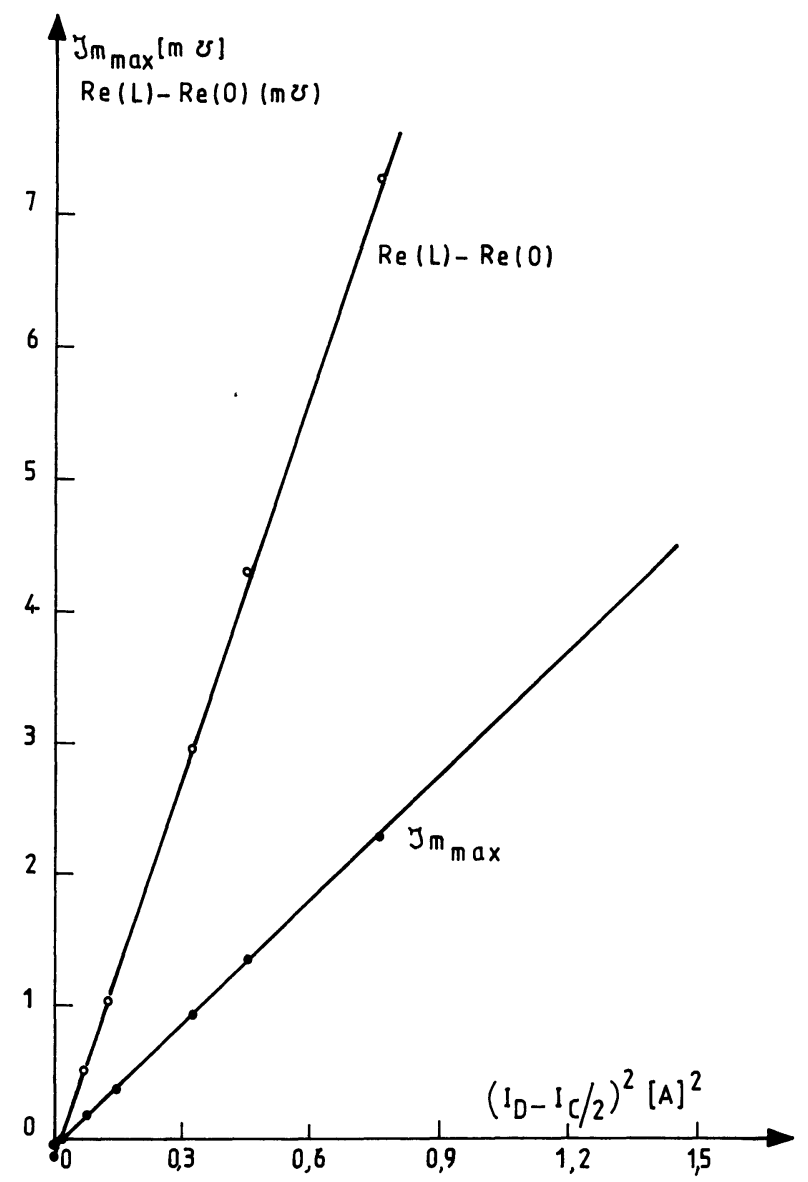

Fig. 9. - Variations expérimentales des quantités $\operatorname{Re}(\mathrm{L})-\operatorname{Re}(0)$ et $\mathrm{Jm} \max$ en fonction de $\left(I_{\mathrm{D}}-I_{\mathrm{C}} / 2\right)^{2}$.

[Experimental variations of $\operatorname{Re}(\mathrm{L})-\operatorname{Re}(0)$ and $\mathrm{Jm}$ max as a function of $\left.\left(I_{\mathrm{D}}-I_{\mathrm{C}} / 2\right)^{2}.\right]$

4.2 DÉTERMINATION DE L'IMPÉDANCE THERMIQUE ET DU SCHÉMA ÉQUIVALENT ÉLECTRIQUE DE L'IMPÉDANCE DE SORTIE DU TRANSISTOR. - i) Une application immédiate de la relaxation de l'admittance de sortie est la détermination de l'impédance thermique complexe. Le principe du tracé d'impédance thermique est basé sur les mesures d'une part de $Y(\omega)$ pour des conditions de courant et tension drain fixées et d'autre part de $k, I_{\mathrm{C}}$ et $\operatorname{Re}(\mathrm{L})$. La transformation géométrique suivante :

$$
Z_{\mathrm{th}}(\omega)=-\frac{Y(\omega)-\operatorname{Re}(\mathrm{L})}{k \cdot I_{\mathrm{D}} \cdot\left(I_{\mathrm{D}}-I_{\mathrm{C}}\right)}
$$

fournit expérimentalement comme l'indique la figure 10 le diagramme complexe de l'impédance thermique. L'amplitude de ce diagramme, sur l'axe réel, est égale à la résistance thermique. 


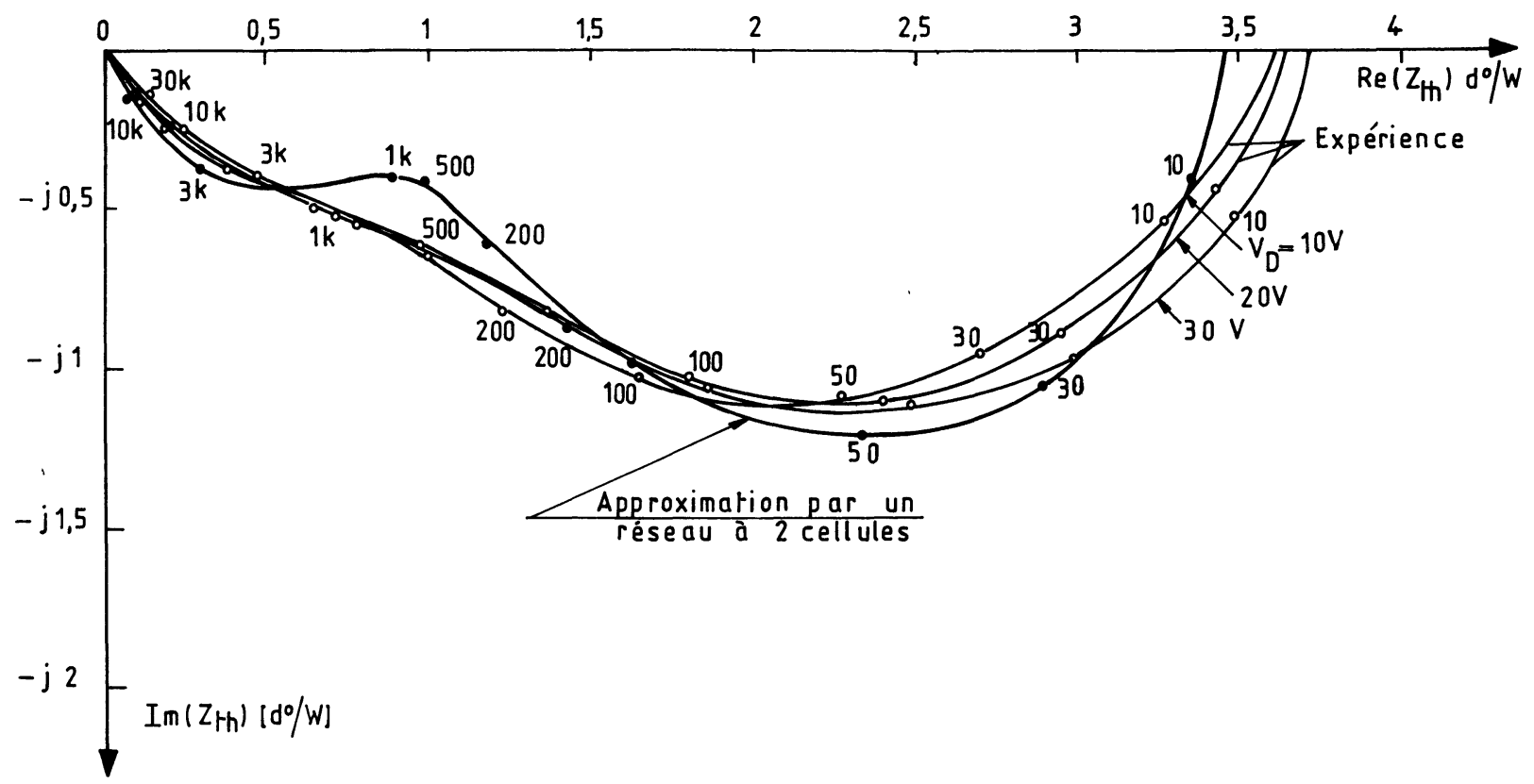

Fig. 10. - Diagramme d'impédance thermique. La fréquence est le paramètre.

[Thermal imṕedance diagram with frequency as parameter.]

Nous avons pu vérifier que les valeurs des résistances thermiques ainsi obtenues sont identiques à celles fournies par le relevé direct du profil de température à la surface du semiconducteur [10].

Ensuite, il est possible de trouver un circuit équivalent à cette impédance sous forme d'association de cellules résistances thermiques-capacités thermiques. Dans le cas des transistors étudiés, une représentation au $1^{\mathrm{er}}$ ordre est proposée sur la figure 11 sous la forme d'un circuit à deux cellules $R_{t h 1}-C_{t h 1}$ et $\mathrm{R}_{\mathrm{th} 2}-\mathrm{C}_{\mathrm{th} 2}$. Ce circuit permet de représenter avec une précision voisine de $10 \%$ les variations fréquentielles de l'impédance thermique comme cela est montré sur la figure 10 où l'on a comparé l'impédance de ce circuit équivalent au diagramme d'impédance thermique relevé expérimentalement.

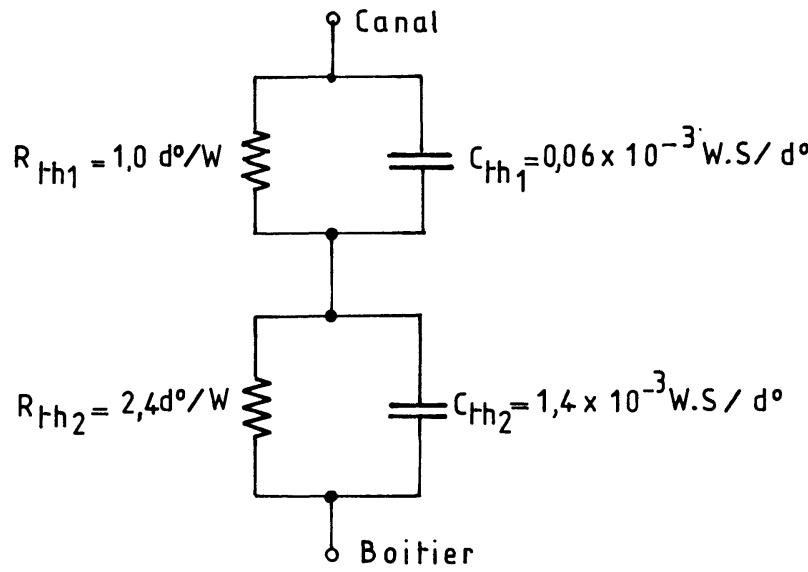

Fig. 11. - Schéma équivalent thermique des transistors étudiés. [Thermal equivalent circuit for the studied devices.] ii) D'un point de vue électrique, l'impédance de sortie du transistor est constituée par la mise en parallèle de l'impédance de sortie d'un transistor idéal dans lequel il n'y aurait pas d'effet thermique et d'une impédance égale à $\left(Z_{\mathrm{th}}(\omega) \cdot I_{\mathrm{D}} \cdot \frac{\partial I_{\mathrm{D}}}{\partial T}\right)^{-1}$ (Fig. 12a).

Cette dernière impédance peut, dans le cas des transistors étudiés, être représentée par la mise en parallèle de cellules résistance-inductance (Fig. 12b).

a)
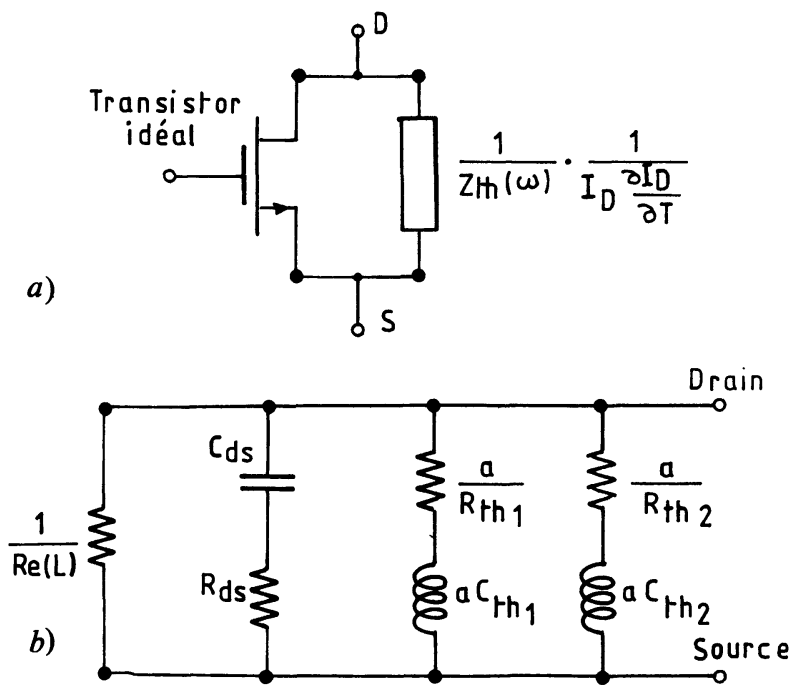

Fig. 12. - Schéma équivalent électrique de l'impédance de sortie ;

$$
a=\left[I_{\mathrm{D}} \cdot \frac{\partial I_{\mathrm{D}}}{\partial T}\right]^{-1}
$$

[Electrical equivalent circuit of the output impedance;

$$
a=\left[I_{\mathrm{D}} \cdot \frac{\partial I_{\mathrm{D}}}{\partial T}\right]^{-1} .
$$


Ces éléments sont positifs si le courant est inférieur au courant critique $I_{\mathrm{C}}$ et dans le cas contraire ils sont négatifs. Quant au transistor idéal, rappelons que son impédance de sortie est représentée par la mise en parallèle de la résistance $\operatorname{Re}(\mathrm{L})^{-1}$ et du circuit résistance $\mathrm{Rds}$-capacité $\mathrm{Cds}$ en série. La capacité Cds est la capacité de transition de la diode drainsubstrat, polarisée en inverse par la tension $V_{\mathrm{D}}$, et la résistance $\mathrm{Rds}$ représente la somme des résistances, entre les contacts de drain et de substrat, du matériau semiconducteur constituant le drain, et le substrat [13].

Ainsi, on peut expliquer les évolutions dans tout le domaine fréquentiel - depuis la fréquence nulle jusqu'aux UHF - de l'admittance de sortie : la figure 13 représente dans le plan de Bode un tel type d'évolution, typique du comportement des transistors à canal court :

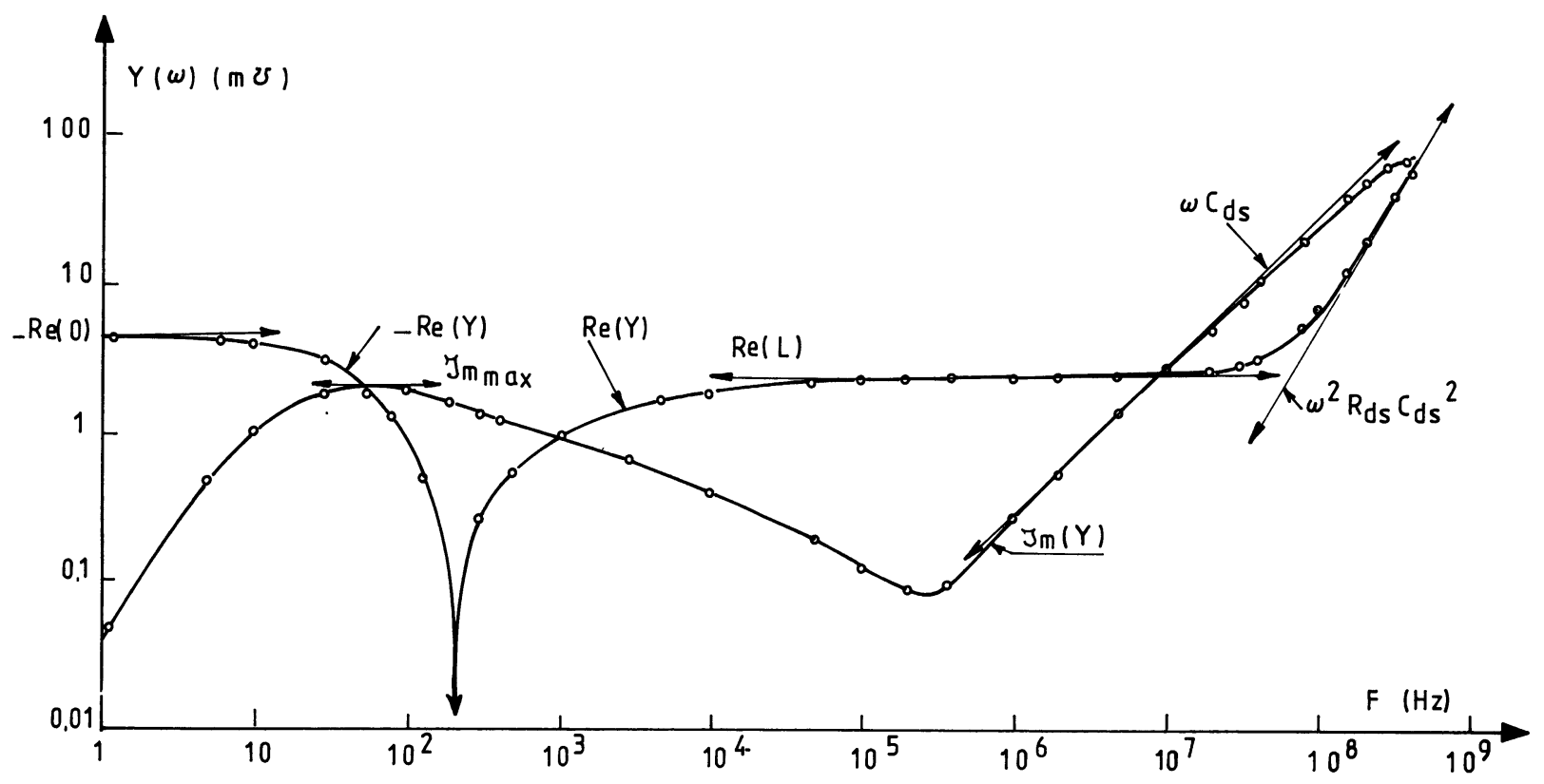

Fig. 13. - Variations fréquentielles des parties réelles et imaginaires de l'admittance de sortie. Gammes $\mathrm{BF}, \mathrm{HF}, \mathrm{VHF}\left(V_{\mathrm{D}}=12 \mathrm{~V}\right.$, $\left.I_{\mathrm{D}}=1 \mathrm{~A}\right)$.

[Real and imaginary parts of the output admittance in the LF, HF and VHF ranges $\left(V_{\mathrm{D}}=12 \mathrm{~V}, I_{\mathrm{D}}=1 \mathrm{~A}\right)$.]

- En régime de basses fréquences $(f<100 \mathrm{kHz})$, les mécanismes qui imposent la valeur de l'admittance sont les effets thermiques et le mécanisme de raccourcissement de canal $(\operatorname{Re}(\mathrm{L}))$ selon les lois que nous venons d'établir.

Dans la gamme des hautes fréquences

$$
(100 \mathrm{kHz}<f<10 \mathrm{MHz})
$$

la partie réelle reste constante, égale à $\operatorname{Re}(\mathrm{L})$, et la partie imaginaire lui est très inférieure. Seul, le mécanisme électrostatique dû au drain impose la résistance de sortie.

- En régime des très hautes fréquences $(f>10 \mathrm{MHz})$ c'est l'élément parasite, lié à la configuration géométrique de la diode de drain, c'est-à-dire à l'existence de la capacité Cds en série avec la résistance Rds, qui impose le mode de croissance proportionnel à la fréquence, pour la partie imaginaire ( $j \mathrm{Cds} \omega$ ), et proportionnel au carré de la fréquence, pour la partie réelle $\left(\operatorname{Rds} \operatorname{Cds}^{2} \omega^{2}\right)$.

5. Conclusion. - Il a été montré dans cet article comment l'effet de contre-réaction thermique dans les transistors MOS, agit sur les propriétés de l'admittance de sortie dans la gamme des basses fréquences, gamme dans laquelle se situent les inverses des constantes de temps thermiques de ces composants. Expérimentalement, cet effet se traduit par l'existence d'un diagramme complexe d'admittance dont les parties réelles et imaginaires peuvent être positives ou négatives et dépendent des conditions de polarisation continues.

La formulation de l'admittance a été établie et fait intervenir l'impédance thermique complexe, le coefficient de température du courant de drain et la résistance de saturation en régime de hautes fréquences. Celle-ci nous a conduit à proposer une méthode expérimentale de détermination de l'impédance thermique ainsi qu'un schéma électrique équivalent du transistor MOS vu de ses électrodes de drain et de source : l'impédance de sortie est constituée par la mise en parallèle de l'impédance de sortie du transistor idéal dans lequel l'effet de couplage électrique-thermique n'est pas pris en compte, et d'un ensemble de cellules résistance-inductance à valeurs positives ou négatives, qui traduisent ce couplage. Dans ce schéma électrique équivalent, le seul élément dont les propriétés sont encore mal connues, pour les MOST à canal court, est la résistance de sortie liée à l'effet de rac- 
courcissement du canal au droit du drain. En effet, à cause de la configuration géométrique particulière de type $\mathrm{N}^{-} \mathrm{N}^{++}$, que présente la diode de drain dans la plupart des dispositifs D MOS ou V MOS à canal court, les analyses classiques visant à décrire les propriétés de cette résistance ne sont pas applicables. Les mécanismes physiques d'injection de porteurs dans la zone $\mathrm{N}^{-}$, d'extension des charges d'espace dans le drain [12] et de multiplication par ionisation sous l'effet du champ électrique élevé [14], généralement négligés dans les transistors classiques, seront à prendre en compte. L'étude que nous avons effectuée fait clairement ressortir que cette résistance de sortie ne pourra être caractérisée que dans un domaine très précis de fréquences, situé hors de la gamme où la relaxation BF intervient et hors de celle où l'influence de la capacité drain-substrat se manifeste.

\section{Bibliographie}

[1] RedDI, V. G. K., SAH, C. T., IEEE Trans. Electron Devices March (1965) 139-141.

[2] Frohman-Bentchkowsky, D., Grove, A. S., IEEE Trans. Electron Devices ED 19 (1969) 108-113.

[3] Merkel, G., Borel, J., CuPCEA, N. Z., IEEE Trans. Electron Devices ED 19 (1972) 681-690.

[4] BauM, G., Solid-State Electron. 13 (1970) 789-798.

[5] Rossel, P., Martinot, H., VAssiliefF, G., Solid State Electron. 19 (1976) 51-56.

[6] LUONG MO DANG, Solid State Electron. 20 (1977) 781-788.

[7] Sharma, D., Gautier, J., Merkel, G., IEEE J. Solid-State Circuits, SC 13 (1978) 378-380.

[8] Müller, O., Pest, J., IEEE Trans. Electron Devices, ED 17 (1970) 770-782.

[9] Newell, W. E., Transient Thermal Analysis of Solid State Power Devices. IEEE Power Electronics Specialists Conference, Pittsburg (1975).
[10] Walshak, L. G., Poole, W. E., Microwave J., February (1977) 62-65.

[11] Heiman, F. P., Miller, H. S., IEEE Trans. Electron Devices March (1965) 142-148.

[12] Combs, S. R., D'Avanzo, D. C., Dutton, R. W., Characterization and Modelling of Simultaneously Fabricated $D M O S$ and $V M O S$ Transistors (IEDM Washington) 1977, 569-572.

[13] OAkes, J. G., Wickstron, R. A., Tremere, D. A. HENG, T. M. S., IEEE Trans. Microwave Theory Tech. MTT 24 (1976) 305-311.

[14] Martinot, H., Rossel, P., Electron. Lett. 7 (1971) 118-120.

[15] Coen, R. W., Muller, R. S., Velocity of Surface Carriers in Inversion. Layers of Silicon. A paraître. 